\title{
Hydrogen Molecule Ion in Strong Magnetic Fields*
}

\author{
C. P. de Melo and R. Ferreira \\ Departamento de Física, Universidade Federal de Pernambuco, 50.000 Recife, Pernambuco, Brazil \\ and \\ H. S. Brandi \\ Departamento de Física, Pontifícia Universidade Católica, 20.000 Rio de Janeiro ZC. 19, Brazil \\ and \\ L. C. M. Miranda \\ Departamento de Física, Universidade de Brasítia, 70.000 Brasîtia, Distrito Federal, Brazil
}

(Received 25 February 1976)

\begin{abstract}
A variational principle is used to determine the binding energy and the fundamental vibrational frequency of $\mathrm{H}_{2}{ }^{+}$in the presence of magnetic fields of strengths found in some neutron stars. It is shown that for $B \sim 10^{12} \mathrm{G}$ the vibrational frequency lies in the ultraviolet or soft-x-ray part of the spectrum.
\end{abstract}

In this Letter we investigate how the molecular vibrations are affected by the presence of intense magnetic fields, considering as an example the simplest molecule, namely, the hydrogen molecule ion. In contrast to the atomic case,$^{1-4}$ the number of papers $\mathrm{s}^{5-7}$ dealing with molecules in strong magnetic fields is relatively small. Nevertheless, as pointed out by Ruderman, ${ }^{2}$ Chen, Ruderman, and Suther land, ${ }^{7}$ and Kadomtsev and Kudryavtsev, ${ }^{5}$ the presence of strong magnetic fields makes possible the formation of molecules of a highly extraordinary type, in which the electron cloud forms a negatively charged "needle," with the nuclei lying on its axis. In particular, it has been shown ${ }^{5}$ that for very strong magnetic fields $\left(B \sim 10^{12}-10^{13} \mathrm{G}\right.$ ) the binding energy of a diatomic molecule is considerably larger than the binding energy of an isolated atom. Intuitively, one should expect a great increase in the vibrational frequency with an increasing field: As a consequence of the magnetic confinement of the needleshaped electron cloud with an increasing field, the nuclei come closer to one another; at the same time, the binding energy becomes larger, leading to an increase in the vibrational frequency of the electronic ground state.

In fairly large magnetic fields, the electron of $\mathrm{H}_{2}{ }^{+}$is expected to move around in circles in the lowest Landau level. This suggests that we should choose our trial function as Landau orbitals with the same center. Let us consider $\mathrm{H}_{2}{ }^{+}$ with the nuclei lying on a single magnetic field line at a distance $R=2 a$ from one another. In cylindrical coordinates $(\rho, \varphi, z)$ with the $z$ axis parallel to the magnetic field, the Schrödinger equation for the electron motion at fixed internuclear distance is

$$
\left\{-\frac{\hbar^{2}}{2 m}\left[\frac{1}{\rho} \frac{\partial}{\partial \rho}\left(\rho \frac{\partial}{\partial \rho}\right)+\frac{\partial^{2}}{\partial z^{2}}+\frac{1}{\rho^{2}} \frac{\partial^{2}}{\partial \varphi^{2}}\right]+\frac{e B}{2 m c} L_{z}+\frac{e^{2} B^{2}}{8 m c^{2}} \rho^{2}-e^{2}\left(\frac{1}{\Gamma_{A}}+\frac{1}{\Gamma_{B}}\right)+\frac{e^{2}}{2 a}\right\} \Psi=E \Psi,
$$

where $L_{z}$ is the $z$ component of the angular momentum, and $r_{A}$ and $r_{B}$ are the distances between the electron and the nuclei $A(\rho=0, z=a)$ and $B$ $(\rho=0, z=-a)$, respectively. It is assumed that all the spins are lined up because of the magnetic field, so that the Zeeman term in Eq. (1) may be neglected.

The reasoning above regarding the shape of the electron cloud and the fact that the electron moves in the lowest Landau orbital suggest a product wave function between a Landau orbital $\Phi_{n=0, \nu=0}(\rho, \varphi)$ and a function $f(z)$ describing the motion along the $z$ axis. The function $\Phi_{n=0, \nu=0}(\rho, \varphi)$ is given by ${ }^{8}$

$$
\Phi_{n=0, \nu=0}(\rho, \varphi)=\exp \left(-\rho^{2} / 4 \rho_{0}{ }^{2}\right) /\left(2 \pi \rho_{0}{ }^{2}\right)^{1 / 2},
$$

where $\rho_{0}=\left(\hbar / m \omega_{c}\right)^{1 / 2}$ and $\omega_{c}=e B / m c$. For the function $f(z)$ we assume

$$
f(z)=\exp \left(-z^{2} / 2 \sigma^{2}\right) /(\sigma \sqrt{\pi})^{1 / 2},
$$

where $\sigma$ is the variational parameter to be determined by minimizing the total energy. This choice for $f(z)$ is similar to that used by Yafet, Keyes, and Adams ${ }^{9}$ in treating the hydrogen atom in strong magnetic fields and has the advantage 
TABLE I. Wave-function parameters, binding energy, and equilibrium internuclear separation of $\mathrm{H}_{2}{ }^{+}$for $10^{9}$ $\leqslant B \leqslant 10^{14} \mathrm{G}$.

\begin{tabular}{ccccc}
\hline $\begin{array}{c}B \\
(\mathrm{G})\end{array}$ & $\alpha$ & $r$ & $\begin{array}{c}E \\
(\mathrm{Ry})\end{array}$ & $\begin{array}{c}R \\
(\AA)\end{array}$ \\
\hline $1 \times 10^{9}$ & 0.8 & 0.8 & -1.03 & 1.831 \\
$5 \times 10^{9}$ & 1.0 & 0.9 & -2.11 & 0.916 \\
$1 \times 10^{10}$ & 1.0 & 0.9 & -2.83 & 0.653 \\
$5 \times 10^{10}$ & 1.5 & 1.2 & -5.53 & 0.390 \\
$1 \times 10^{11}$ & 1.8 & 1.4 & -7.27 & 0.320 \\
$5 \times 10^{11}$ & 2.6 & 1.8 & -13.18 & 0.186 \\
$1 \times 10^{12}$ & 3.2 & 2.1 & -16.76 & 0.151 \\
$5 \times 10^{12}$ & 4.9 & 2.9 & -28.15 & 0.095 \\
$1 \times 10^{13}$ & 6.1 & 3.5 & -34.63 & 0.079 \\
$5 \times 10^{13}$ & 10.0 & 5.3 & -53.80 & 0.055 \\
$1 \times 10^{14}$ & 12.4 & 6.3 & -63.71 & 0.045 \\
\hline \hline
\end{tabular}

over a $e^{-|z|}$ function that its derivative is continuous everywhere. Using $\Psi=\Phi_{n=0, \nu=0}(\rho, \varphi) f(z)$ and introducing the following dimensionless parameters,

$$
\alpha=\sigma / 2 \rho_{0}, \quad \Gamma=a_{0} / \rho_{0} \sqrt{2},
$$

the total energy $E(\sigma, a)=\langle\Psi|\hat{\mathcal{H}}| \Psi\rangle$ can then be written as

$$
E=\epsilon(\alpha, r) E_{H} / \lambda^{2},
$$

where $E_{H}=e^{2} / 2 a_{0}{ }^{2}=13.6 \mathrm{eV}, a_{0}$ is the Bohr radi-

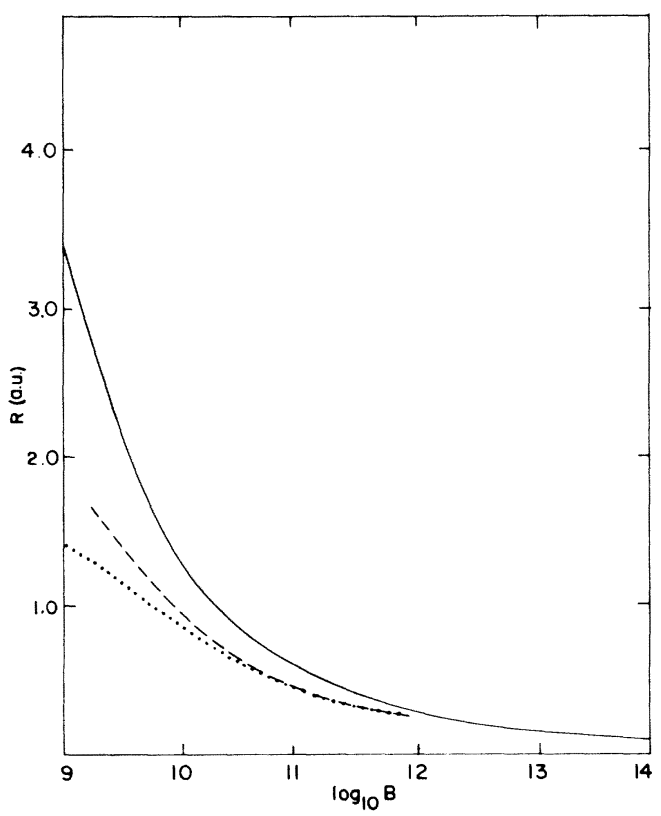

FIG. 1. Equilibrium internuclear separation of $\mathrm{H}_{2}{ }^{+}$ as a function of the magnetic field. The full line is the result of the present theory whereas the dotted and the dashed lines are the mean radius of the hydrogen atoms as given by Refs. 8 and 9 , respectively. us, $\lambda=\rho_{0} / a_{0}$, and

$$
\epsilon(\alpha, r)=1+\frac{1}{8 \alpha^{2}}+\frac{\lambda}{r \sqrt{2}}-\frac{4 \lambda}{\alpha \sqrt{\pi}} F(\alpha, r) .
$$

$F(\alpha, r)$ is the Coulomb attraction overlap and is given by

$$
\begin{aligned}
& F(\alpha, r) \\
& =\int_{0}^{\infty} d y y \exp \left(-y^{2}\right) \int_{-\infty}^{+\infty} d x \frac{\exp \left(-x^{2} / 2 \alpha^{2}\right)}{\left[y^{2}+(x-r)^{2}\right]^{1 / 2}},
\end{aligned}
$$

$\alpha$ and $2 r$ describe the variational parameter $\sigma$ and the internuclear distance in units of the cyclotron radius $\rho_{0}$, respectively, whereas $\lambda$ is a measure of the cyclotron radius in units of the Bohr radius.

We can now minimize the total energy as a function of $\alpha$. Since the function $F(\alpha, r)$ has no simple analytical solution this was accomplished numerically, by varying $\alpha$ and obtaining for a given $r$ the value of $\alpha$ which minimizes Eq. (7). The values of $\alpha$ and $r$ which minimize the energy and the equilibrium internuclear separation are presented in Table $I$ for values of $B$ in the range $10^{9} \mathrm{G}<B<10^{14} \mathrm{G}$. The curves show results for fields smaller than $10^{10} \mathrm{G}$, and it is clear that for those values of fields the curve in Fig. 4 presents

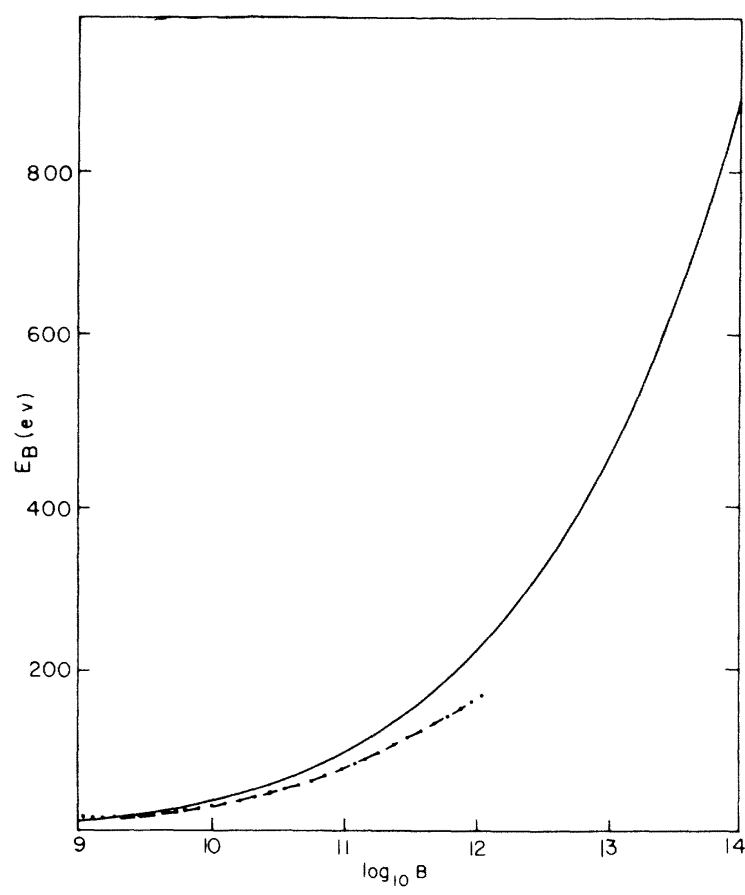

FIG. 2. The binding energy of $\mathrm{H}_{2}{ }^{+}$as a function of the magnetic field is represented by the full line. The dotted and dashed lines are the binding energy of the hydrogen atom as given by Refs.9 and 10, respectively. 


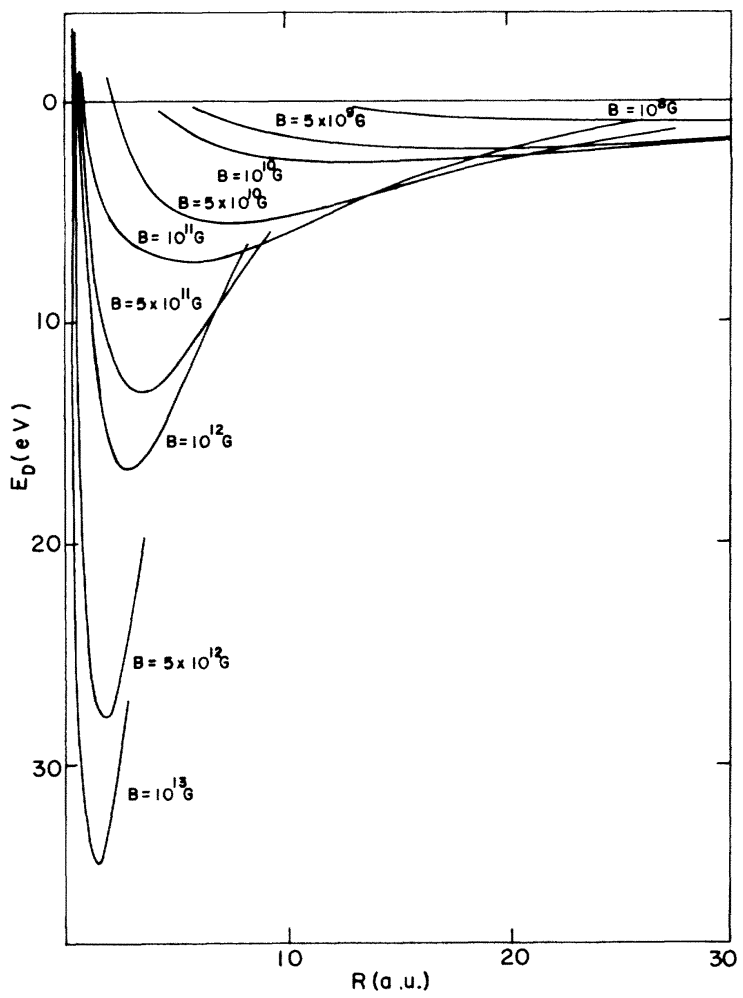

FIG. 3. Dissociation energy versus internuclear separation for $\mathrm{H}_{2}^{+}$.

no minimum. As predicted by Kadomtsev and Kudryavtsev, ${ }^{5}$ the binding energy of a diatomic molecule in strong fields is considerably larger than the binding energy of isolated atoms. The effects of confinement and stabilization of the molecule can be seen in Figs. 1 and 2, where we plot the equilibrium internuclear distance and the binding energy $E_{B}$ as a function of $B$, respectively. The dissociation energy $E_{D}$ is defined as the difference in energy between energy of the molecule and that of a proton plus one hydrogen atom at infinite distance (but in the same $B$ ): $E_{B}{ }^{\text {molecule }}=E_{D}$ $+E_{B}{ }^{\text {atom }}$. These values for the equilibrium internuclear distance and binding energy should be compared with the carresponding ones of $\mathrm{H}_{2}{ }^{+}$in the absence of the external magnetic fields: equilibrium internuclear separation of 2.0 a.u. and binding energy of $16.38 \mathrm{eV}$. The confinement and stabilization of the $\mathrm{H}_{2}{ }^{+}$become even more apparent from Fig. 3, where we plot the dissociation energy curve for several values of $B$. It follows from Fig. 3 that, as the field increases, the potential curves become sharper and deeper leading to a great increase in the vibrational frequency of the molecule. In fact, using the harmonic approximation for the bottom of the potential curves

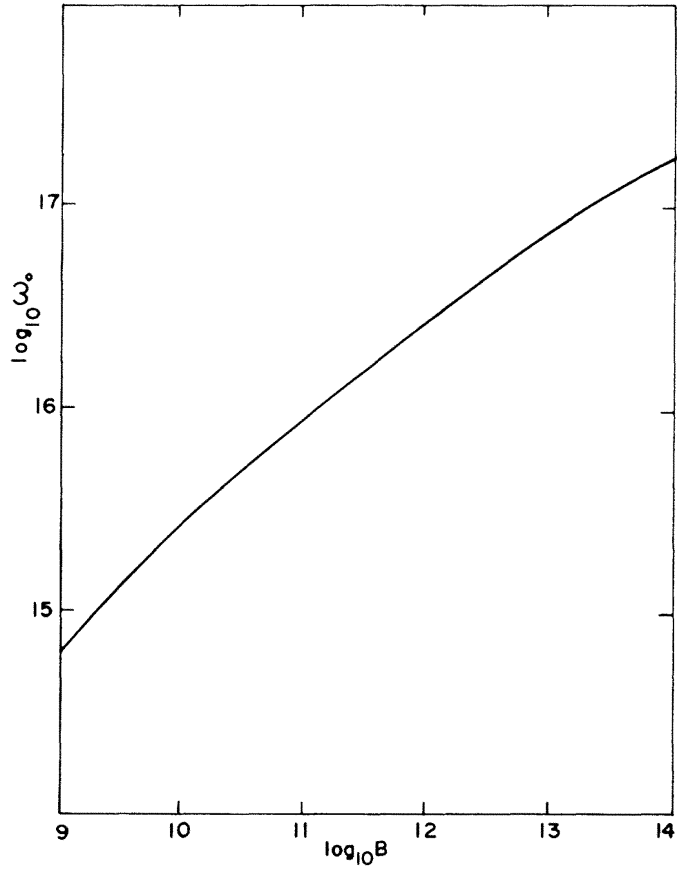

FIG. 4. Fundamental vibrational frequency of $\mathrm{H}_{2}{ }^{+}$as a function of the magnetic field.

close to the equilibrium distance, we can evaluate the fundamental vibrational frequency $\omega_{0}$, namely $\omega_{0}=(k / \mu)^{1 / 2}$ where $\mu$ is the reduced mass of the system and $k$ is given by

$$
k=2\left[E(R)-E\left(R_{\mathrm{min}}\right)\right] /\left(R-R_{\mathrm{min}}\right)^{2} .
$$

The plot of $\omega_{0}$ as a function of $B$ is presented in Fig. 4. As predicted, the fundamental vibrational frequency increases with increasing field. In particular, for $B \sim 10^{12}-10^{13} \mathrm{G}, \omega_{0}$ lies in the ultraviolet and soft $\mathrm{x}$-ray range.

In conclusion it should be emphasized that our model contains a number of limitations. Nevertheless, the essential results confirm what one expects qualitatively. Among the limitations, the present theory is valid for $10^{10} \mathrm{G} \lesssim B \lesssim 10^{14} \mathrm{G}$. One can see this by regarding $\mathrm{H}_{2}{ }^{+}$in the ground state as a bounded particle of mass $\mu$ (the reduced mass) and energy $E_{B}$ (the binding energy). Hence, as in the hydrogen atom case, the strong field limit is determined by requiring the cyclotron precession of such a particle to be larger than $E_{B}$. This, in turn, restricts our theory to fields greater than $10^{10} \mathrm{G}$. Consequently our wave function is not appropriate for $B<10^{10} \mathrm{G}$, for which a linear-combination-of-atomic-orbitals treatment based on hydrogenlike wave functions would be more adequate. ${ }^{11}$ Furthermore, for fields much larger than $10^{12} \mathrm{G}$ one can no 
longer neglect relativistic corrections to the Landau orbitals. From the condition $\hbar \omega_{c}=m c^{2}$ the upper limit for the magnetic field is estimated to be of the order of $4.14 \times 10^{13} \mathrm{G}$.

*Work partially supported by the Brasilian Agencies Conselho Nacional de Pesquisas, Coordenacão do Aperfeicoamento de Pessoal de Nivel Superior, and Banco Nacional do Desanvolvemento Economico.

${ }^{1}$ R. Cohen, J. Lodenquai, and M. Ruderman, Phys. Rev. Lett. 25, 467 (1970).

${ }^{2}$ M. Ruderman, Phys. Rev。Lett. 27, 1306 (1971)。

${ }^{3}$ E. R. Smith, R. J. W. Henry, G. L. Surmelian, R. F.
O'Connell, and A. K. Rajagopal, Phys. Rev. D 6,3700

(1972) [see also Astrophys. J. 190, 741 (1974)].

${ }^{4}$ H. S. Brandi, Phys. Rev. A 11,1835 (1975).

${ }^{5}$ B. B. Kadomtsev and V. S. Kudryavtsev, Pis'ma Zh.

Eksp. Teor. Fiz. 13, 15 (1970) [JETP Lett. 13, 9 (1971)]. ${ }^{6}$ S. T. Chui, Phys. Rev. B 9 , 3438 (1974).

${ }^{7}$ H. H. Chen, M. A. Ruderman, and P. A. Sutherland, Astrophys. J. 191, 473 (1974).

${ }^{8}$ L. D. Landau and E. M. Lifshitz, Quantum Mechanics (Pergamon, Oxford, 1965), p. 421.

${ }^{9}$ Y. Yafet, R. W. Keyes, and E. N. Adams, J. Phys. Chem. Solids 1,137 (1956).

${ }^{10}$ V. Canuto and D. Kelly, Astrophys. Space Sci. 17, 27 (1972).

${ }^{11}$ L. Carvalho and P. de Melo, private communication.

\title{
Energies and Relative Intensities of $K \alpha \alpha \mathbf{X}$-Ray Transitions
}

\author{
A. R. Knudson, K. W. Hill,* P. G. Burkhalter, and D. J. Nagel \\ Naval Research Laboratory, Washington, D. C. 20375
}

(Received 26 May 1976)

\begin{abstract}
$K \alpha \alpha$ absolute energies and energy shifts relative to twice the $K \alpha$ energy have been measured in ion-atom collisions at $\mathrm{MeV}$ energies in the region from $Z=12$ to 26 . For $Z$ $=12$ to 22 good agreement is obtained with Hartree-Fock energy calculations assuming an $E 1$ transition with two $L$-shell vacancies in the initial state. $K \alpha \alpha$ to $K \alpha$ intensity ratios are also presented.
\end{abstract}

The study of transitions in which two electrons both change to more tightly bound inner-shell orbitals and the energy is carried off by a single photon has an interesting history. Such cooperative transitions received extensive theoretical attention in early attempts to understand the energy of $\mathrm{x}$-ray satellite lines, ${ }^{1-3}$ and then were neglected for several decades. The recent observation by Wölfli et al. ${ }^{4}$ of cooperative $K \alpha \alpha$ x-ray transitions (resulting from the filling of two $K$-shell vacancies by two $L$-shell electrons) from $\mathrm{Fe}$ and $\mathrm{Ni}$ in high-energy heavy-ion collisions has caused a resurgence of interest in this area. ${ }^{5-11}$ These transitions provide a new testing ground for determining the adequacy of present calculational methods for obtaining transition energies and rates.

Final configurations associated with the observed $K \alpha \alpha$ transitions were not initially identified by Wölfli et al. ${ }^{4}$ Calculations indicated that the observed energy was in agreement with an $E 1$ transition $\left[(1 s)^{-2} \rightarrow(2 s)^{-1}(2 p)^{-1}\right]$, but the $E 2$ transition $\left[(1 s)^{-2}-(2 p)^{-2}\right]$ was predicted to be dominant ${ }^{8}$ in highly stripped ions. This Letter presents the first extensive comparison of the measured and calculated $K \alpha \alpha$ energies (over the region $12 \leqslant Z \leqslant 26$ ), together with the measured in- tensities of the $K \alpha \alpha$ transition relative to the $K \alpha$ transition.

In the present experiment ions accelerated by a 5-MV Van de Graaff accelerator were used to bombard thick targets. The energy of the ions was generally between 3 and $3.5 \mathrm{MeV}$, although in two cases doubly ionized beams of $7.0 \mathrm{MeV}$ were also used. $X$ rays emitted at $90^{\circ}$ to the incident beam were measured with a $\mathrm{Si}(\mathrm{Li})$ detector of $200-\mathrm{eV}$ resolution at $6 \mathrm{keV}$. In obtaining the $K \alpha \alpha$ spectra, sufficiently thick $\mathrm{Be}$ and $\mathrm{Al}$ absorbers were used to reduce the transmission of the corresponding $K \alpha$ radiation to about $10^{-8}$ in order to avoid any possibility of pulse pileup which might obscure or simulate the $K \alpha \alpha$ line. These absorbers resulted in about $10 \%$ transmission for the $K \alpha \alpha$ radiation. Spectra were also measured with the absorbers removed in order to obtain the yield of $K \alpha \mathrm{x}$ rays. Energy calibrations were obtained by using $\mathrm{Fe}^{55}$ and $\mathrm{Am}^{241}$ sources and also by bombarding various targets with proton and helium beams. When helium beams were used, corrections were made to account for the presence of enhanced satellite lines. ${ }^{12}$

Figure 1 shows the spectrum obtained from 3.5- $\mathrm{MeV} \mathrm{Ar}^{+}$ions incident on a $\mathrm{Ca}$ target. A $0.0076-\mathrm{cm} \mathrm{Al}$ absorber was used in addition to 